\title{
On the Solution of Generalized Space Time Fractional Telegraph Equation
}

\author{
Badr S. Alkahtani, ${ }^{1}$ Vartika Gulati, ${ }^{2}$ and Pranay Goswami ${ }^{3}$ \\ ${ }^{1}$ Mathematics Department, College of Science, King Saud University, P.O. Box 1142, Riyadh 11989, Saudi Arabia \\ ${ }^{2}$ Department of Mathematics, University of Rajasthan, Rajasthan 302055, India \\ ${ }^{3}$ School of Liberal Studies, Ambedkar University Delhi, Delhi 110006, India
}

Correspondence should be addressed to Pranay Goswami; pranaygoswami83@gmail.com

Received 29 May 2015; Accepted 3 August 2015

Academic Editor: Gisele Mophou

Copyright (C) 2015 Badr S. Alkahtani et al. This is an open access article distributed under the Creative Commons Attribution License, which permits unrestricted use, distribution, and reproduction in any medium, provided the original work is properly cited.

We present the solution of generalized space time fractional telegraph equation by using Sumudu variational iteration method which is the combination of variational iteration method and Sumudu transform. We tried to overcome the difficulties in finding the value of Lagrange multiplier by this new technique.

\section{Introduction}

Differential calculus has its great importance in the field of Science and Technology [1]. The partial differential equations are one of the most powerful mathematical tools to describe, define, and explain the various phenomena of physics and chemistry and the process to solve them is a new interesting field to study. Telegraph equations are hyperbolic partial differential equations that gained remarkable interest due to their numerous applications in high-frequency transmission lines, propagation of electrical signals, optimization of guided communication system, and the propagation of quantum particles and many other physical and chemical phenomena. They are also applicable to designing high-voltage transmission lines.

Recently generalized space time fractional telegraph equations were used to describe number of chemical and physical phenomena with remarkably interesting results. The solutions of generalized space time fractional telegraph equations were obtained by the various numerical methods. Numerous investigations have been done to solve fractional telegraph equations. The methods, namely, generalized differential transform, Fourier-Laplace transform, separations of variables, perturbation theory, and basic variational iteration, have been used to investigate the solution of homogeneous and nonhomogenous time fractional telegraph equation and the solutions are given in the form of Mittag-Leffler functions [2-6]. Nowadays variational iteration method is being used to solve many fractional differential equations and the presence of Sumudu transform is making the process rich.

In this paper we modified the variational iteration method (VIM) by making use of the Sumudu transform and apply it to generalized space time fractional telegraph equations. This Sumudu variational iteration method enables us to overcome the difficulties that arise in finding the general Lagrange multipliers.

\section{Mathematical Preliminaries}

Definition 1. The Caputo fractional derivative of order $\alpha>0$ is defined as [7]

$$
D_{t}^{\alpha} f(t)= \begin{cases}\frac{\partial^{\alpha} f(t)}{\partial t^{\alpha}}=\frac{1}{\Gamma(m-\alpha)} \int_{0}^{t} \frac{1}{(t-\tau)^{\alpha-m+1}} \frac{\partial^{m} f(\tau)}{\partial \tau^{m}} d \tau, & m-1<\alpha \leq m, m \in N, \\ \frac{\partial^{m} f}{\partial t^{m}}, & \text { if } \alpha=m \in N,\end{cases}
$$


where ${ }_{0} D_{t}^{\alpha}$ is called the Caputo derivative operator. By using Definition 1, we have the following result:

$$
\begin{aligned}
& D_{t}^{\alpha}\left(t^{\beta}\right) \\
& = \begin{cases}\frac{\Gamma(\beta+1)}{\Gamma(\beta-\alpha+1)} t^{\beta-\alpha}, & m-1<\alpha \leq m, \beta>m-1, \beta \in \mathfrak{R}, \\
0 & m-1<\alpha \leq m, \beta \leq m-1 .\end{cases}
\end{aligned}
$$

Definition 2. For $n-1<\alpha<n, 0 \leq \beta \leq 1, n \in N$, the generalized Hilfer fractional derivative $[8,9]$ is defined by

$$
\left(D_{a \pm}^{\alpha, \beta} f\right)(t)=\left( \pm I_{t}^{\beta(n-\alpha)} \frac{d}{d t}\left(I_{t}^{(1-\beta)(n-\alpha)} f\right)\right)(t) .
$$

In the definition above, type $\beta$ allows $\left(D_{a \pm}^{\alpha, \beta}\right)$ to interpolate continuously between the classical Riemann-Liouville fractional derivative and the Caputo fractional derivative. Clearly, the case, $\beta=0$, reverts back to yield the classical RiemannLiouville fractional derivative:

$$
D_{a+}^{\alpha, 0} f(t)=D^{n} I_{t}^{(n-\alpha)} f(t)=D_{t}^{\alpha} f(t), \quad 0<\alpha<1 .
$$

Furthermore, in the case $\beta=1$, the Caputo fractional derivative is obtained:

$$
D_{a+}^{\alpha, 1} f(t)=I_{t}^{(n-\alpha)} D^{n} f(t)=D_{t}^{\alpha} f(t), \quad 0<\alpha<1 .
$$

Definition 3. The Sumudu transform [10-12] over the set of functions

$$
\begin{aligned}
A & =\left\{f(t)\left|\exists M, \tau_{1}, \tau_{2}>0,\right| f(t) \mid<M e^{|t| / \tau_{i}}, \text { if } t\right. \\
& \left.\in(-1)^{j} \times[0, \infty)\right\}
\end{aligned}
$$

is defined as

$$
G(u)=S[f(t)]=\int_{0}^{\infty} f(u t) e^{-t} d t, \quad u \in\left(-\tau_{1}, \tau_{2}\right) .
$$

And the Sumudu transform of generalized Hilfer fractional derivative $\left(D_{a \pm}^{\alpha, \beta} y\right)(x)$ is given as follows:

$$
\begin{aligned}
S( & \left.D_{a+}^{\alpha, \beta} y\right)(t) \\
= & u^{-\alpha} S[y(t)](u) \\
& \quad-\sum_{k=0}^{n-1}\left[u^{k-n+\beta(n-\alpha)} \lim _{t \rightarrow a+} \frac{d^{k}}{d t^{k}}\left(I_{a+}^{(n-\alpha)(1-\beta)} y\right)(t)\right] .
\end{aligned}
$$

Definition 4. The two-parameter Mittag-Leffler function $E_{\alpha}(z)$ is defined [13] as

$$
E_{\alpha, \beta}(z)=\sum_{k=0}^{\infty} \frac{z^{k}}{\Gamma(\alpha k+\beta)},
$$

where $\alpha, \beta \in \mathbb{C} ; \mathfrak{R}(\alpha)>0 ; \mathfrak{R}(\beta)>0 ; z \in \mathbb{C}$.

By using Definition 4 , we have

(1) $E_{2,1}\left(z^{2}\right)=\cosh z$,

(2) $E_{2,2}\left(z^{2}\right)=(\sinh z) / z$.
Definition 5. A generalization of Mittag-Leffler function $E_{\alpha, \beta}(z)$ of (9) is as follows [14]:

$$
E_{\alpha, \beta}^{\gamma}(z)=\sum_{k=0}^{\infty} \frac{(\gamma)_{k}}{\Gamma(\alpha k+\beta)} \frac{z^{k}}{k !}
$$

where $\alpha, \beta, \gamma \in \mathbb{C} ; \mathfrak{R}(\alpha)>0 ; \mathfrak{R}(\beta)>0 ; z \in \mathbb{C}$ and $\gamma_{k}$ denotes the familiar Pochhammer symbol or the shifted factorial, since

$$
\begin{aligned}
&(1)_{k}=k ! \quad\left(k \in N_{0}\right), \\
&(\gamma)_{k}=\frac{\Gamma(\gamma+k)}{\Gamma(\gamma)}(k=0 ; \gamma \in C \backslash\{0\}) \\
&= \begin{cases}1 & (k \in N ; \gamma \in C) \\
\gamma(\gamma+1) \cdots(\gamma+k-1) & \left(N_{0}=N \cup\{0\}=\{0,1,2, \ldots\}\right) .\end{cases}
\end{aligned}
$$

\section{Variational Iteration Method}

The analytical technique used by many researchers to study linear and nonlinear partial differential equations is commonly known as the variational iteration method. This technique of variational iteration was developed by Chinese mathematician $\mathrm{He}$ [15]. To illustrate the basic idea of this method, we consider the general nonlinear system of the following form:

$$
L u(x, t)+N u(x, t)=f(x, t),
$$

where $u$ is the unknown function, $L$ and $N$ are linear and nonlinear operators, respectively, and $f$ is the source term.

The basic character of the method is to construct the following correction functional for (12):

$$
\begin{aligned}
u_{n+1}(x, t) & \\
= & u_{n}(x, t) \\
& \quad+\int_{0}^{x} \lambda\left[L u_{n}(\xi, t)+N u_{n}(\xi, t)-f(\xi, t)\right] d \xi
\end{aligned}
$$

where $\lambda$ is called the general Lagrange multiplier [16] and $u_{n}$ is the approximate solution of $n$th order. If we have seen the whole process of the Lagrange multipliers in the case of an algebraic equation then solution of the algebraic equation $f(x)=0$ can be obtained by an iteration formula:

$$
x_{n+1}=x_{n}+\lambda f\left(x_{n}\right) .
$$

The optimality condition for the extreme $\delta x_{n+1} / \delta x_{n}=0$ leads to

$$
\lambda=-\frac{1}{f^{\prime}\left(x_{n}\right)},
$$

where $\delta$ is the classical variational operator. 


\section{Sumudu Variational Iteration Method (SVIM)}

Consider the following generalized space time fractional telegraph equation:

$$
\begin{aligned}
D_{0+}^{\alpha, \alpha_{1}} v(x, t)= & a_{1} \frac{\partial^{\beta} v}{\partial t^{\beta}}(x, t)+a_{2} \frac{\partial^{\gamma} v}{\partial t^{\gamma}}(x, t)+a_{3} v(x, t) \\
& +f(x, t),
\end{aligned}
$$

where $n-1<\alpha<n ; 0<\alpha_{1} \leq 1 ; \beta \leq 2 ; 0<\gamma \leq 1 ; x, t \geq 0$; $\partial^{\beta} / \partial t^{\beta}$ and $\partial^{\gamma} / \partial t^{\gamma}$ are Caputo derivatives defined by (1) and $a_{1}, a_{2}, a_{3}$ are constants.

Applying Sumudu transform with respect to $x$ in both sides of (16),

$$
\begin{aligned}
& S\left[D_{0+}^{\alpha, \alpha_{1}} v(x, t)\right]=S\left[a_{1} \frac{\partial^{\beta} v}{\partial t^{\beta}}(x, t)+a_{2} \frac{\partial^{\gamma} v}{\partial t^{\gamma}}(x, t)\right. \\
& \left.+a_{3} v(x, t)+f(x, t)\right] .
\end{aligned}
$$

Using (8) with $a=0$ we get

$$
\begin{aligned}
& u^{-\alpha} \bar{v}(u, t)-\sum_{k=0}^{m-1}\left[u^{k-n+\alpha_{1}(n-\alpha)} I_{0+}^{(n-\alpha)\left(1-\alpha_{1}\right)} v^{k}(0, t)\right] \\
& -S\left[a_{1} \frac{\partial^{\beta} v}{\partial t^{\beta}}(x, t)+a_{2} \frac{\partial^{\gamma} v}{\partial t^{\gamma}}(x, t)+a_{3} v(x, t)\right. \\
& +f(x, t)]=0 .
\end{aligned}
$$

By the formula of (14), we get the iteration formula for the above as follows:

$$
\begin{aligned}
& \bar{v}_{n+1}(u, t)=\bar{v}_{n}(u, t)+\lambda\left[u^{-\alpha} \bar{v}_{n}(u, t)\right. \\
& -\sum_{k=0}^{m-1}\left[u^{k-n+\alpha_{1}(n-\alpha)} I_{0+}^{(n-\alpha)\left(1-\alpha_{1}\right)} v^{k}(0, t)\right] \\
& \quad-S\left(a_{1} \frac{\partial^{\beta} v_{n}}{\partial t^{\beta}}(x, t)+a_{2} \frac{\partial^{\gamma} v_{n}}{\partial t^{\gamma}}(x, t)+a_{3} v_{n}(x, t)\right. \\
& \quad+f(x, t))] .
\end{aligned}
$$

Putting $\lambda=-u^{\alpha}$, the Lagrange multiplier in (19) becomes

$$
\begin{aligned}
& \bar{v}_{n+1}(u, t)=\left[u^{\alpha} \sum_{k=0}^{m-1}\left[u^{k-n+\alpha_{1}(n-\alpha)} I_{0+}^{(n-\alpha)\left(1-\alpha_{1}\right)} v^{k}(0, t)\right]\right. \\
& +u^{\alpha} S\left(a_{1} \frac{\partial^{\beta} v_{n}}{\partial t^{\beta}}(x, t)+a_{2} \frac{\partial^{\gamma} v_{n}}{\partial t^{\gamma}}(x, t)+a_{3} v_{n}(x, t)\right. \\
& +f(x, t))] .
\end{aligned}
$$

Now taking inverse Sumudu transform on both sides of (20),

$$
\begin{aligned}
& v_{n+1}(u, t) \\
& \quad=S^{-1}\left[u^{\alpha} \sum_{k=0}^{m-1}\left[u^{k-n+\alpha_{1}(n-\alpha)} I_{0+}^{(n-\alpha)\left(1-\alpha_{1}\right)} v^{k}(0, t)\right]\right. \\
& +u^{\alpha} S\left(a_{1} \frac{\partial^{\beta} v_{n}}{\partial t^{\beta}}(x, t)+a_{2} \frac{\partial^{\gamma} v_{n}}{\partial t^{\gamma}}(x, t)+a_{3} v_{n}(x, t)\right. \\
& \quad+f(x, t))] .
\end{aligned}
$$

This is the iteration formula for solving generalized space time fractional telegraph equation with the following initial iteration:

$$
\begin{aligned}
v_{0} & (x, t) \\
& =S^{-1}\left(\sum_{k=0}^{m-1}\left[u^{k-n+\alpha_{1}(n-\alpha)+\alpha} I_{0+}^{(n-\alpha)\left(1-\alpha_{1}\right)} v^{k}(0, t)\right]\right) .
\end{aligned}
$$

\section{Main Results}

Theorem 6. Consider the space time fractional homogenous telegraph equation

$$
\begin{aligned}
& D_{0+}^{\alpha, \alpha_{1}} v(x, t)=\frac{\partial^{2} v}{\partial t^{2}}(x, t)+\frac{\partial v}{\partial t}(x, t)+v(x, t), \\
& x, t \geq 0,1<\alpha \leq 2,0<\alpha_{1} \leq 1,
\end{aligned}
$$

with initial conditions

$$
\begin{gathered}
I_{0+}^{(1-\alpha)\left(1-\alpha_{1}\right)} v(0, t)=e^{-t}, \\
I_{0+}^{(2-\alpha)\left(1-\alpha_{1}\right)} v^{\prime}(0, t)=e^{-t},
\end{gathered}
$$

has the solution in closed form as

$$
\begin{aligned}
& v(x, t)=\lim _{n \rightarrow \infty} v_{n}(x, t) \\
& =e^{-t} x^{\alpha_{1}(1-\alpha)+\alpha-1}\left[E_{\alpha, \alpha_{1}(1-\alpha)+\alpha}\left(x^{\alpha}\right)\right. \\
& \left.+x^{\alpha_{1}} E_{\alpha, \alpha_{1}(2-\alpha)+\alpha}\left(x^{\alpha}\right)\right] .
\end{aligned}
$$

Proof. We have the following space time fractional homogenous equation:

$$
D_{0+}^{\alpha, \alpha_{1}} v(x, t)=\frac{\partial^{2} v}{\partial t^{2}}(x, t)+\frac{\partial v}{\partial t}(x, t)+v(x, t) .
$$

By using iteration formula (21) derived above, we get the following iteration for (26):

$$
\begin{aligned}
& v_{n+1}(x, t) \\
&=S^{-1}\left[\sum_{k=0}^{m-1}\left[u^{k-n+\alpha_{1}(n-\alpha)+\alpha} I_{0+}^{(n-\alpha)\left(1-\alpha_{1}\right)} v^{k}(0, t)\right]\right. \\
&\left.+u^{\alpha} S\left(\frac{\partial^{2} v_{n}}{\partial t^{2}}(x, t)+\frac{\partial v_{n}}{\partial t}(x, t)+v_{n}(x, t)\right)\right] .
\end{aligned}
$$


The initial iteration is

$$
\begin{aligned}
& v_{0}(x, t) \\
& =S^{-1}\left(\sum_{k=0}^{m-1}\left[u^{k-n+\alpha_{1}(n-\alpha)+\alpha} I_{0+}^{(n-\alpha)\left(1-\alpha_{1}\right)} v^{k}(0, t)\right]\right), \\
& v_{0}(x, t)=S^{-1}\left[u^{-1+\alpha_{1}(1-\alpha)+\alpha} e^{-t}+u^{-1+\alpha_{1}(2-\alpha)+\alpha} e^{-t}\right], \\
& v_{0}(x, t) \\
& \quad=\frac{x^{\alpha_{1}(1-\alpha)+\alpha-1}}{\Gamma\left(\alpha_{1}(1-\alpha)+\alpha\right)} e^{-t}+\frac{x^{\alpha_{1}(2-\alpha)+\alpha-1}}{\Gamma\left(\alpha_{1}(2-\alpha)+\alpha\right)} e^{-t} .
\end{aligned}
$$

Now by putting the values of " $n$ " we get the iterations. For $v_{1}(x, t)$ put $n=0$ in (27); we find

$$
\begin{aligned}
& v_{1}(x, t)=S^{-1}\left[u^{-1+\alpha_{1}(1-\alpha)+\alpha} e^{-t}+u^{-1+\alpha_{1}(2-\alpha)+\alpha} e^{-t}\right. \\
& \left.+u^{\alpha} S\left(\frac{\partial^{2} v_{0}}{\partial t^{2}}(x, t)+\frac{\partial v_{0}}{\partial t}(x, t)+v_{0}(x, t)\right)\right], \\
& v_{1}(x, t)=S^{-1}\left[u^{-1+\alpha_{1}(1-\alpha)+\alpha} e^{-t}+u^{-1+\alpha_{1}(2-\alpha)+\alpha} e^{-t}\right. \\
& +u^{\alpha} S\left(\frac{x^{\alpha_{1}(1-\alpha)+\alpha-1}}{\Gamma\left(\alpha_{1}(1-\alpha)+\alpha\right)} e^{-t}\right. \\
& \left.\left.+\frac{x^{\alpha_{1}(2-\alpha)+\alpha-1}}{\Gamma\left(\alpha_{1}(2-\alpha)+\alpha\right)} e^{-t}\right)\right], \\
& v_{1}(x, t)=S^{-1}\left[u^{-1+\alpha_{1}(1-\alpha)+\alpha} e^{-t}+u^{-1+\alpha_{1}(2-\alpha)+\alpha} e^{-t}\right. \\
& \left.+u^{-1+\alpha_{1}(1-\alpha)+2 \alpha} e^{-t}+u^{-1+\alpha_{1}(2-\alpha)+2 \alpha} e^{-t}\right] .
\end{aligned}
$$

Taking inverse Sumudu transform of (31) we get

$$
\begin{aligned}
v_{1}(x, t)= & \frac{x^{\alpha+\alpha_{1}(1-\alpha)-1}}{\Gamma\left(\alpha+\alpha_{1}(1-\alpha)\right)} e^{-t} \\
& +\frac{x^{\alpha+\alpha_{1}(2-\alpha)-1}}{\Gamma\left(\alpha+\alpha_{1}(2-\alpha)\right)} e^{-t} \\
& +\frac{x^{2 \alpha+\alpha_{1}(1-\alpha)-1}}{\Gamma\left(2 \alpha+\alpha_{1}(1-\alpha)\right)} e^{-t} \\
& +\frac{x^{2 \alpha+\alpha_{1}(2-\alpha)-1}}{\Gamma\left(2 \alpha+\alpha_{1}(2-\alpha)\right)} e^{-t} .
\end{aligned}
$$

For $n=1, v_{2}(x, t)$ is given by the following iteration:

$$
\begin{aligned}
& v_{2}(x, t)=S^{-1}\left[u^{-1+\alpha_{1}(1-\alpha)+\alpha} e^{-t}+u^{-1+\alpha_{1}(2-\alpha)+\alpha} e^{-t}\right. \\
& \left.+u^{\alpha} S\left(\frac{\partial^{2} v_{1}}{\partial t^{2}}(x, t)+\frac{\partial v_{1}}{\partial t}(x, t)+v_{1}(x, t)\right)\right], \\
& v_{2}(x, t)=\frac{x^{\alpha+\alpha_{1}(1-\alpha)-1}}{\Gamma\left(\alpha+\alpha_{1}(1-\alpha)\right)} e^{-t}+\frac{x^{\alpha+\alpha_{1}(2-\alpha)-1}}{\Gamma\left(\alpha+\alpha_{1}(2-\alpha)\right)} \\
& \cdot e^{-t}+\frac{x^{2 \alpha+\alpha_{1}(1-\alpha)-1}}{\Gamma\left(2 \alpha+\alpha_{1}(1-\alpha)\right)} e^{-t} \\
& +\frac{x^{2 \alpha+\alpha_{1}(2-\alpha)-1}}{\Gamma\left(2 \alpha+\alpha_{1}(2-\alpha)\right)} e^{-t}+\frac{x^{3 \alpha+\alpha_{1}(1-\alpha)-1}}{\Gamma\left(3 \alpha+\alpha_{1}(1-\alpha)\right)} e^{-t} \\
& +\frac{x^{3 \alpha+\alpha_{1}(2-\alpha)-1}}{\Gamma\left(3 \alpha+\alpha_{1}(2-\alpha)\right)} e^{-t} \cdot
\end{aligned}
$$

Then the general term in successive approximation is given by

$$
\begin{aligned}
& v_{n}(x, t) \\
& =e^{-t} \sum_{k=0}^{n} x^{(k+1) \alpha+\alpha_{1}(1-\alpha)-1}\left[\frac{1}{\Gamma\left((k+1) \alpha+\alpha_{1}(1-\alpha)\right)}\right. \\
& \left.+\frac{x^{\alpha_{1}}}{\Gamma\left((k+1) \alpha+\alpha_{1}(2-\alpha)\right)}\right] .
\end{aligned}
$$

The solution in a closed form is given as follows:

$$
\begin{aligned}
& v(x, t)=\lim _{n \rightarrow \infty} v_{n}(x, t) \\
& =e^{-t} x^{\alpha_{1}(1-\alpha)+\alpha-1}\left[E_{\alpha, \alpha_{1}(1-\alpha)+\alpha}\left(x^{\alpha}\right)\right. \\
& \left.+x^{\alpha_{1}} E_{\alpha, \alpha_{1}(2-\alpha)+\alpha}\left(x^{\alpha}\right)\right] .
\end{aligned}
$$

Theorem 7. Consider the following generalized space time fractional nonhomogenous telegraph equation

$$
\begin{aligned}
D_{0+}^{\alpha, \alpha_{1}} v(x, t)= & \frac{\partial^{2} v}{\partial t^{2}}(x, t)+\frac{\partial v^{\gamma}}{\partial t^{\gamma}}(x, t)+v(x, t)-x^{2} \\
& -t+1, \\
1< & \alpha \leq 2,0<\gamma \leq 1,0<\alpha_{1} \leq 1 x, t \geq 0,
\end{aligned}
$$

with initial conditions

$$
\begin{gathered}
I_{0}^{(1-\alpha)\left(1-\alpha_{1}\right)} v(0, t)=t \\
I_{0}^{(2-\alpha)\left(1-\alpha_{1}\right)} v_{x}(0, t)=0
\end{gathered}
$$


has the solution in closed form as

$$
\begin{aligned}
& v(x, t)=\lim _{n \rightarrow \infty} v_{n}(x, t)=x^{2}\left[1-2 E_{\alpha, 3}\left(x^{\alpha}\right)\right] \\
& +\left(1-t+\frac{t^{1-\gamma}}{\Gamma(2-\gamma)}\right)\left[E_{\alpha, 1}\left(x^{\alpha}\right)-1\right] \\
& +t x^{\alpha_{1}(1-\alpha)+\alpha-1} E_{\alpha, \alpha_{1}(1-\alpha)+\alpha}\left(x^{\alpha}\right) \\
& +\left[x^{\alpha_{1}(1-\alpha)+\alpha-1} E_{\alpha, \alpha_{1}(1-\alpha)+\alpha}^{1}\left(x^{\alpha}\right)-E_{\alpha, 1}^{1}\left(x^{\alpha}\right)\right] \\
& \cdot \frac{t^{1-\gamma}}{\Gamma(2-\gamma)} .
\end{aligned}
$$

Proof. We have the following space time fractional nonhomogenous telegraph equation:

$$
\begin{aligned}
D_{0+}^{\alpha, \alpha_{1}}(x, t)= & \frac{\partial^{2} v}{\partial t^{2}}(x, t)+\frac{\partial v^{\gamma}}{\partial t^{\gamma}}(x, t)+v(x, t)-x^{2}-t \\
& +1 .
\end{aligned}
$$

By using iteration formula (21) derived above, we get the following iteration for (39):

$$
\begin{aligned}
& v_{n+1}(x, t) \\
& =S^{-1}\left[\sum_{k=0}^{m-1}\left[u^{k-n+\alpha_{1}(n-\alpha)+\alpha} I_{0+}^{(n-\alpha)\left(1-\alpha_{1}\right)} v^{k}(0, t)\right]\right. \\
& +u^{\alpha} S\left(\frac{\partial^{2} v_{n}}{\partial t^{2}}(x, t)+\frac{\partial^{\gamma} v_{n}}{\partial t^{\gamma}}(x, t)+v_{n}(x, t)-x^{2}\right. \\
& -t+1)] .
\end{aligned}
$$

The initial iteration $v_{0}(x, t)$ is given by

$$
\begin{aligned}
& v_{0}(x, t) \\
& \quad=S^{-1}\left(\sum_{k=0}^{m-1}\left[u^{k-n+\alpha_{1}(n-\alpha)+\alpha} I_{0+}^{(n-\alpha)\left(1-\alpha_{1}\right)} v^{k}(0, t)\right]\right), \\
& v_{0}(x, t)=S^{-1}\left[u^{-1+\alpha_{1}(1-\alpha)+\alpha} I_{0+}^{(1-\alpha)\left(1-\alpha_{1}\right)} v(0, t)\right] .
\end{aligned}
$$

Using initial conditions (37), we get

$$
v_{0}(x, t)=S^{-1}\left[u^{-1+\alpha_{1}(1-\alpha)+\alpha} t\right] .
$$

Taking inverse Sumudu transform we find the initial iteration as

$$
v_{0}(x, t)=\frac{x^{\alpha_{1}(1-\alpha)+\alpha-1}}{\Gamma\left(\alpha_{1}(1-\alpha)+\alpha\right)} t .
$$

Now by putting the values of " $n$ " we get the iterations. For $v_{1}(x, t)$ put $n=0$ in (40); we have

$$
\begin{gathered}
v_{1}(x, t)=S^{-1}\left[u^{-1+\alpha_{1}(1-\alpha)+\alpha} t+u^{\alpha} S\left(\frac{\partial^{2} v_{0}}{\partial t^{2}}(x, t)\right.\right. \\
\left.\left.+\frac{\partial^{\gamma} v_{0}}{\partial t^{\gamma}}(x, t)+v_{0}(x, t)-x^{2}-t+1\right)\right] .
\end{gathered}
$$

Taking Sumudu transform after solving the derivative terms we get

$$
\begin{aligned}
& v_{1}(x, t)=S^{-1}\left[u^{\alpha_{1}(1-\alpha)+\alpha-1} t+u^{\alpha_{1}(1-\alpha)+2 \alpha-1} \frac{t^{1-\gamma}}{\Gamma(2-\gamma)}\right. \\
& \left.+u^{\alpha_{1}(1-\alpha)+2 \alpha-1} t-2 u^{2+\alpha}-t u^{\alpha}+u^{\alpha}\right] .
\end{aligned}
$$

Applying inverse Sumudu transform,

$$
\begin{aligned}
& v_{1}(x, t)=\frac{x^{\alpha_{1}(1-\alpha)+\alpha-1}}{\Gamma\left(\alpha_{1}(1-\alpha)+\alpha\right)} t \\
& +\frac{x^{\alpha_{1}(1-\alpha)+2 \alpha-1}}{\Gamma\left(\alpha_{1}(1-\alpha)+2 \alpha\right)} \frac{t^{1-\gamma}}{\Gamma(2-\gamma)} \\
& +\frac{x^{\alpha_{1}(1-\alpha)+2 \alpha-1}}{\Gamma\left(\alpha_{1}(1-\alpha)+2 \alpha\right)} t-2 \frac{x^{\alpha+2}}{\Gamma(\alpha+3)} \\
& -\frac{x^{\alpha}}{\Gamma(\alpha+1)} t+\frac{x^{\alpha}}{\Gamma(\alpha+1)}, \\
& v_{2}(x, t)=\frac{x^{\alpha_{1}(1-\alpha)+\alpha-1}}{\Gamma\left(\alpha_{1}(1-\alpha)+\alpha\right)} t \\
& +\frac{x^{\alpha_{1}(1-\alpha)+2 \alpha-1}}{\Gamma\left(\alpha_{1}(1-\alpha)+2 \alpha\right)} \frac{t^{1-\gamma}}{\Gamma(2-\gamma)} \\
& -\frac{x^{2 \alpha}}{\Gamma(2 \alpha+1)} t-\frac{x^{\alpha}}{\Gamma(\alpha+1)} t \\
& +\frac{x^{\alpha_{1}(1-\alpha)+2 \alpha-1}}{\Gamma\left(\alpha_{1}(1-\alpha)+2 \alpha\right)} t \\
& +\frac{x^{\alpha_{1}(1-\alpha)+3 \alpha-1}}{\Gamma\left(\alpha_{1}(1-\alpha)+3 \alpha\right)} t \\
& +2 \frac{x^{\alpha_{1}(1-\alpha)+3 \alpha-1}}{\Gamma\left(\alpha_{1}(1-\alpha)+3 \alpha\right)} \frac{t^{1-\gamma}}{\Gamma(2-\gamma)} \\
& -2 \frac{x^{2 \alpha+2}}{\Gamma(2 \alpha+3)}+\frac{x^{2 \alpha}}{\Gamma(2 \alpha+1)} \\
& -2 \frac{x^{\alpha+2}}{\Gamma(\alpha+3)}+\frac{x^{\alpha}}{\Gamma(\alpha+1)} \\
& -\frac{x^{2 \alpha}}{\Gamma(2 \alpha+1)} \frac{t^{1-\gamma}}{\Gamma(2-\gamma)} \text {. }
\end{aligned}
$$

Then the general term in successive approximation is given by

$$
\begin{aligned}
v_{n}(x, t)= & t \frac{x^{\alpha_{1}(1-\alpha)+\alpha-1}}{\Gamma\left(\alpha_{1}(1-\alpha)+\alpha\right)} \\
& +t \sum_{k=1}^{n} \frac{x^{\alpha_{1}(1-\alpha)+(k+1) \alpha-1}}{\Gamma\left(\alpha_{1}(1-\alpha)+(k+1) \alpha\right)}
\end{aligned}
$$




$$
\begin{aligned}
& -2 x^{2} \sum_{k=1}^{n} \frac{x^{k \alpha}}{\Gamma(k \alpha+3)} \\
& +\frac{t^{1-\gamma}}{\Gamma(2-\gamma)} \sum_{k=1}^{n} k \frac{x^{\alpha_{1}(1-\alpha)+(k+1) \alpha-1}}{\Gamma\left(\alpha_{1}(1-\alpha)+(k+1) \alpha\right)} \\
& +(1-t) \sum_{k=1}^{n} \frac{x^{k \alpha}}{\Gamma(k \alpha+1)} \\
& -\frac{t^{1-\gamma}}{\Gamma(2-\gamma)} \sum_{k=1}^{n}(k-1) \frac{x^{k \alpha}}{\Gamma(k \alpha+1)}
\end{aligned}
$$

The solution in a closed form is given by

$$
\begin{aligned}
& v(x, t)=\lim _{n \rightarrow \infty} v_{n}(x, t)=x^{2}\left[1-2 E_{\alpha, 3}\left(x^{\alpha}\right)\right] \\
& +\left(1-t+\frac{t^{1-\gamma}}{\Gamma(2-\gamma)}\right)\left[E_{\alpha, 1}\left(x^{\alpha}\right)-1\right] \\
& +t x^{\alpha_{1}(1-\alpha)+\alpha-1} E_{\alpha, \alpha_{1}(1-\alpha)+\alpha}\left(x^{\alpha}\right) \\
& +\left[x^{\alpha_{1}(1-\alpha)+\alpha-1} E_{\alpha, \alpha_{1}(1-\alpha)+\alpha}^{1}\left(x^{\alpha}\right)-E_{\alpha, 1}^{1}\left(x^{\alpha}\right)\right] \\
& \cdot \frac{t^{1-\gamma}}{\Gamma(2-\gamma)} .
\end{aligned}
$$

\section{Special Cases}

If we take $\alpha_{1}=1$, in above Theorems 6 and 7 , we have the results obtained by Alawad et al. [3].

Corollary 8. The space time fractional homogenous telegraph equation

$$
\begin{array}{r}
\frac{\partial^{\alpha} v}{\partial x^{\alpha}}(x, t)=\frac{\partial^{2} v}{\partial t^{2}}(x, t)+\frac{\partial v}{\partial t}(x, t)+v(x, t), \\
x, t \geq 0,1<\alpha \leq 2,
\end{array}
$$

with the initial conditions

$$
\begin{gathered}
v(0, t)=e^{-t}, \\
v_{k}(0, t)=e^{-t}
\end{gathered}
$$

has the solution in closed form as

$$
v(x, t)=\lim _{n \rightarrow \infty} v(x, t)=e^{-t}\left[E_{\alpha, 1}\left(x^{\alpha}\right)+x E_{\alpha, 2}\left(x^{\alpha}\right)\right] .
$$

Remark 9. If we put $\alpha=2$ in (51) then the following solution is same as that obtained by Wazwaz [17]:

$$
\begin{aligned}
v(x, t) & =e^{-t}\left[E_{2,1}\left(x^{2}\right)+x E_{2,2}\left(x^{2}\right)\right] \\
& =e^{-t}\left[\cosh (x)+x \frac{\sinh (x)}{x}\right]=e^{x-t}
\end{aligned}
$$

Corollary 10. The space time fractional nonhomogenous telegraph equation

$$
\begin{aligned}
\frac{\partial^{\alpha} v}{\partial x^{\alpha}}(x, t)= & \frac{\partial^{2} v}{\partial t^{2}}(x, t)+\frac{\partial v^{\gamma}}{\partial t^{\gamma}}(x, t)+v(x, t)-x^{2}-t \\
& +1, \quad 1<\alpha \leq 2,0<\gamma \leq 1, x, t \geq 0,
\end{aligned}
$$

with initial conditions

$$
\begin{array}{r}
v(0, t)=t, \\
v_{x}(0, t)=0
\end{array}
$$

has the solution as follows:

$$
\begin{aligned}
v(x, t)= & \lim _{n \rightarrow \infty} v_{n}(x, t) \\
= & t+x^{2}\left[1-2 E_{\alpha, 3}\left(x^{\alpha}\right)\right] \\
& +\left(1+\frac{t^{1-\gamma}}{\Gamma(2-\gamma)}\right)\left[E_{\alpha, 1}\left(x^{\alpha}\right)-1\right] .
\end{aligned}
$$

\section{Conflict of Interests}

Authors declare that there is no conflict of interests.

\section{Acknowledgments}

Authors are thankful to the reviewers for their fruitful comments to improve this paper. Dr. Badr S. Alkahtani is also grateful to King Saud University, Deanship of Scientific Research, College of Science Research Center, for supporting this project.

\section{References}

[1] A. A. Kilbas, H. M. Srivastava, and J. J. Trujillo, Theory and Applications of Fractional Differential Equations, Elsevier, Amsterdam, The Netherlands, 2006.

[2] J. Chen, F. Liu, and V. Anh, "Analytical solution for the timefractional telegraph equation by the method of separating variables," Journal of Mathematical Analysis and Applications, vol. 338, no. 2, pp. 1364-1377, 2008.

[3] F. A. Alawad, E. A. Yousif, and A. I. Arbab, "A new technique of Laplace variational iteration method for solving spacetime fractional telegraph equations," International Journal of Differential Equations, vol. 2013, Article ID 256593, 10 pages, 2013.

[4] M. Garg, P. Manohar, and S. L. Kalla, "Generalized differential transform method to space-time fractional telegraph equation," International Journal of Differential Equations, vol. 2011, Article ID 548982, 9 pages, 2011.

[5] Y. Khan, J. Diblík, N. Faraz, and Z. Šmarda, "An efficient new perturbative Laplace method for space-time fractional telegraph equations," Advances in Difference Equations, vol. 2012, article 204, 2012.

[6] A. Sevimlican, "An approximation to solution of space and time fractional telegraph equations by He's variational iteration method," Mathematical Problems in Engineering, vol. 2010, Article ID 290631, 10 pages, 2010. 
[7] M. Caputo, Elasticita e Dissipazione, Zanichelli, Bologna, Italy, 1969.

[8] R. Hilfer, Ed., Applications of Fractional Calculus in Physics, World Scientific, Singapore, 2000.

[9] R. Hilfer, Y. Luchko, and Ž. Tomovski, "Operational method for the solution of fractional differential equations with generalized Riemann-Liouville fractional derivatives," Fractional Calculus and Applied Analysis, vol. 12, no. 3, pp. 299-318, 2009.

[10] M. A. Asiru, "Sumudu transform and the solution of integral equations of convolution type," International Journal of Mathematical Education in Science and Technology, vol. 32, no. 6, pp. 906-910, 2001.

[11] F. B. M. Belagecam and A. A. Karaballi, "Sumudu transform fundamental properties investigations and applications," Journal of Applied Mathematics and Stochastic Analysis, vol. 2006, 23 pages, 2006.

[12] F. B. M. Belgacem, "Introducing and analyzing deeper Sumudu properties," Nonlinear Studies, vol. 13, no. 1, pp. 23-41, 2006.

[13] A. Wiman, "Uber den Fundamental satz in der theoric der Fucntions," Acta Mathematica, vol. 29, pp. 191-201, 1905.

[14] T. R. Prabhakar, "A singular integral equation with a generalized Mittag-Leffler function in the kernel," Yokohama Mathematical Journal, vol. 19, pp. 7-15, 1971.

[15] J.-H. He, "Variational iteration method-a kind of non-linear analytical technique: some examples," International Journal of Non-Linear Mechanics, vol. 34, no. 4, pp. 699-708, 1999.

[16] M. Inokuti, H. Sekine, and T. Mura, "General use of the Lagrange multiplier in nonlinear mathematical physics," in Variational Methods in the Mechanics of Solids, S. Nemat-Nasser, Ed., pp. 156-162, Pergamon Press, New York, NY, USA, 1978.

[17] A.-M. Wazwaz, Partial Differential Equations and Solitary Waves Theory, Nonlinear Physical Science, Higher Education Press, Beijing, China; Springer, Heidelberg, Germany, 2009. 


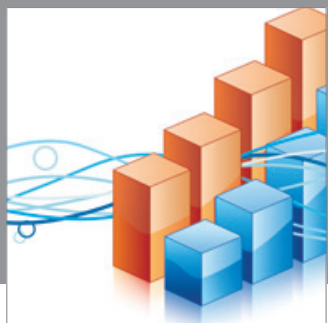

Advances in

Operations Research

mansans

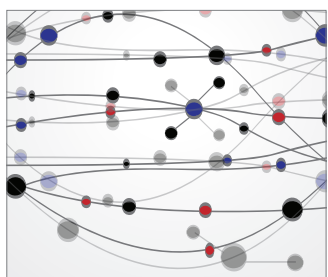

The Scientific World Journal
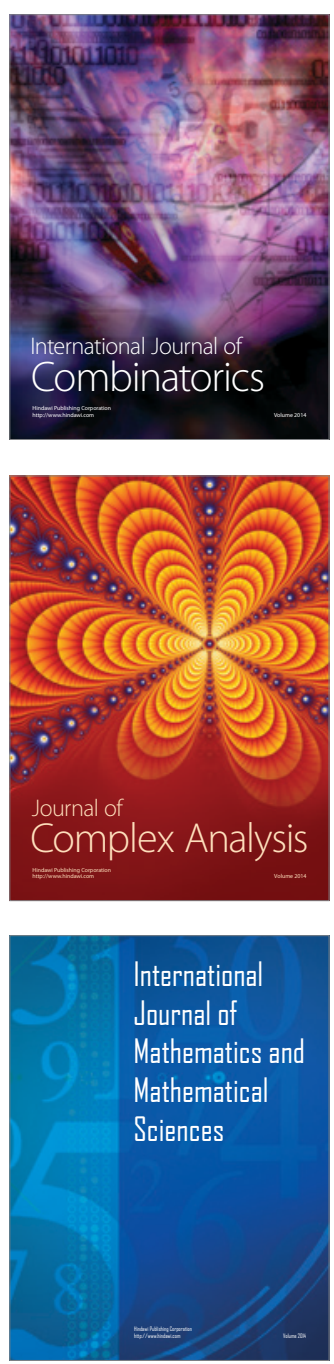
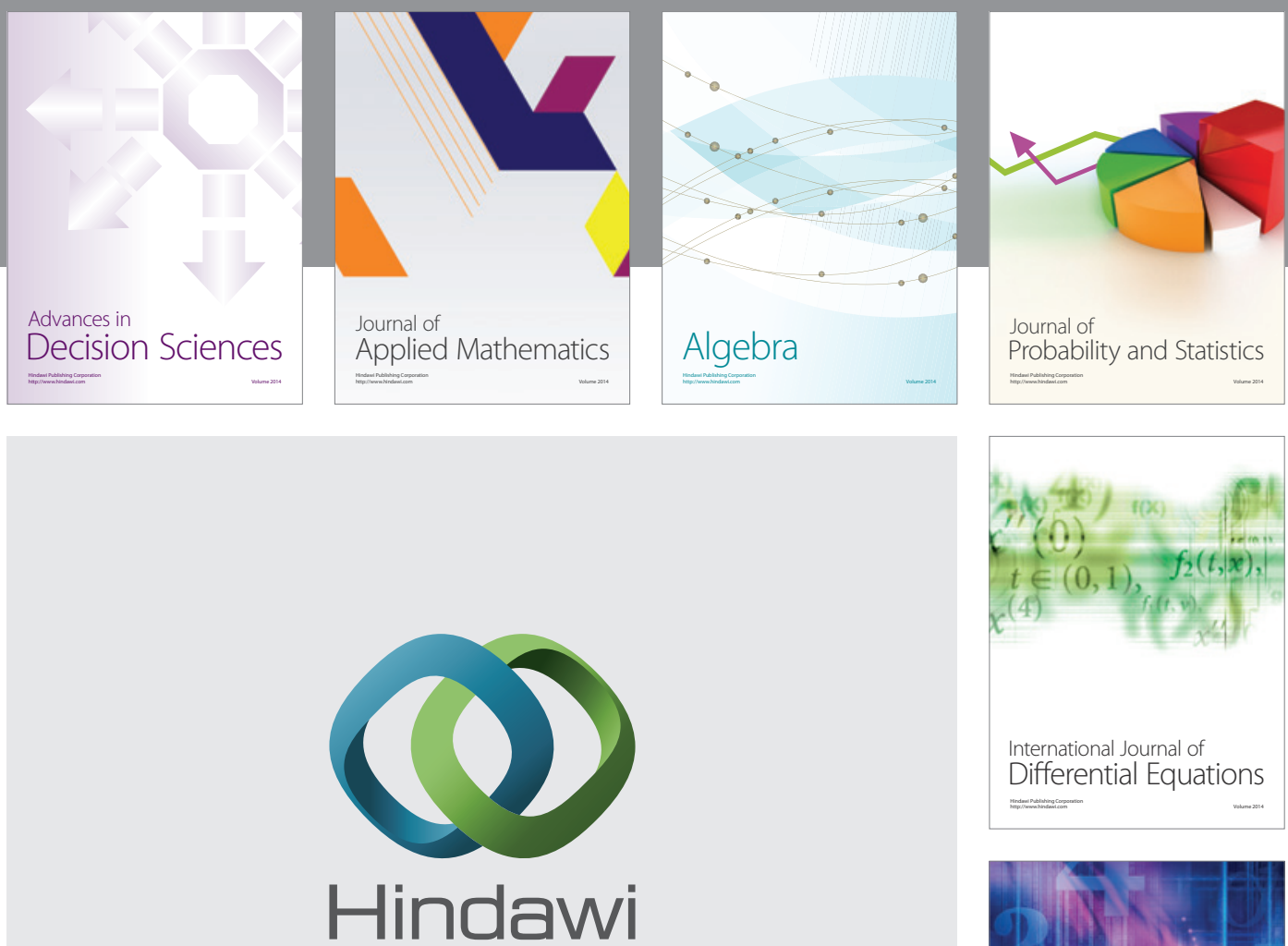

Submit your manuscripts at http://www.hindawi.com
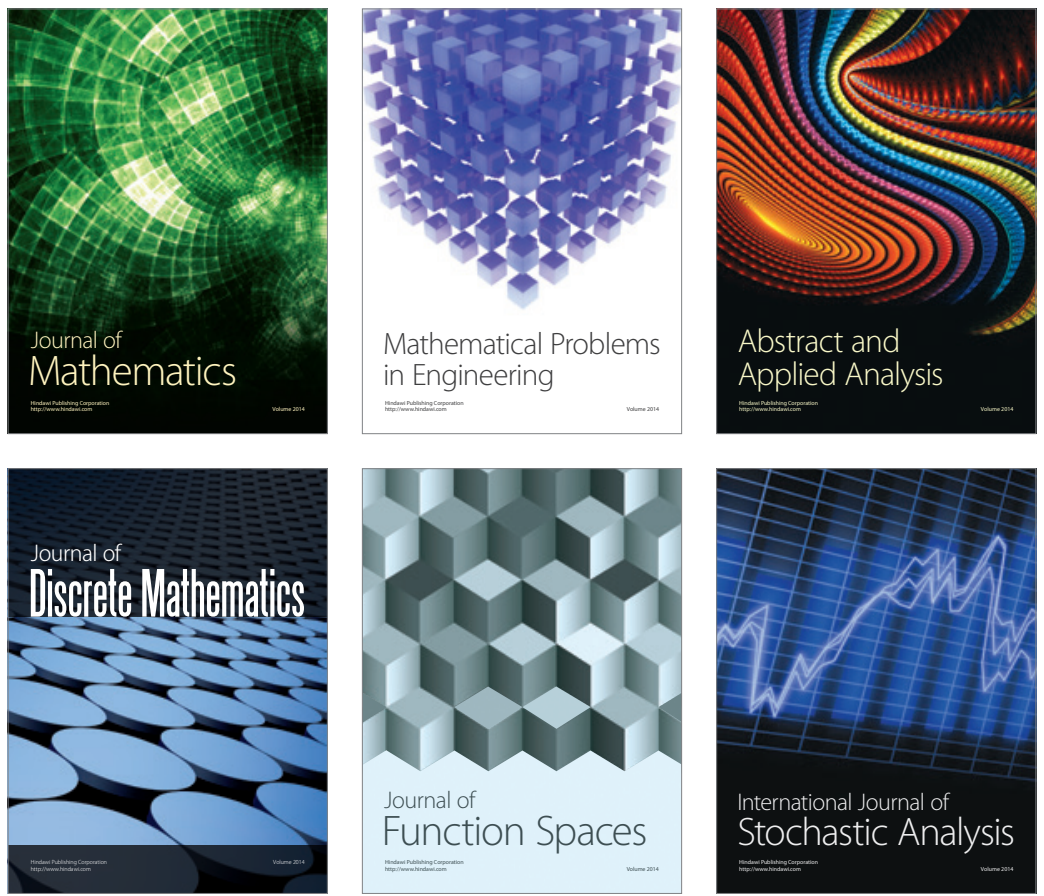

Journal of

Function Spaces

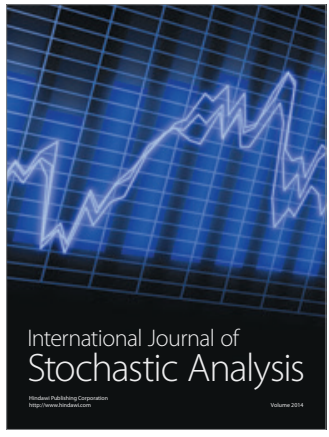

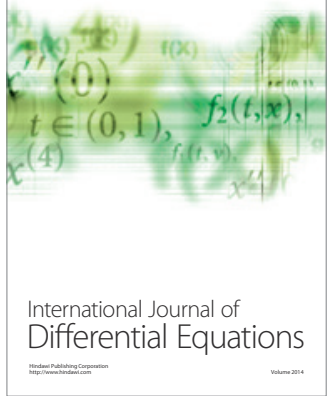
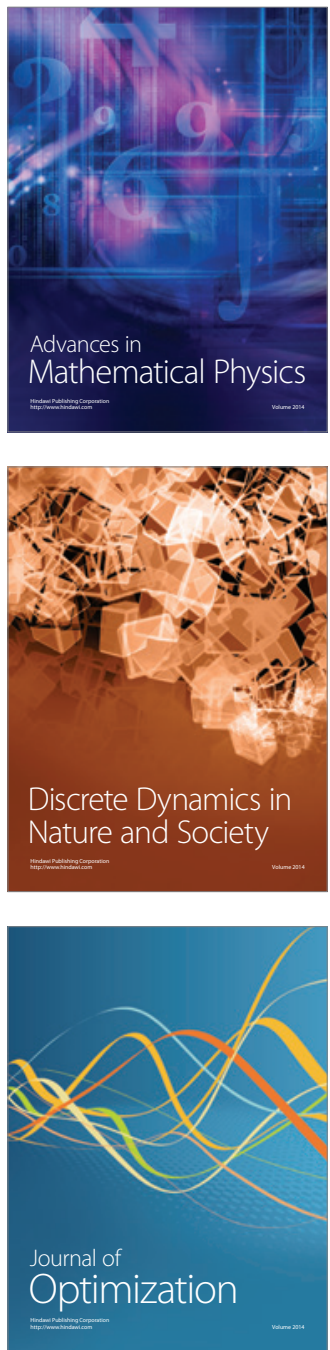